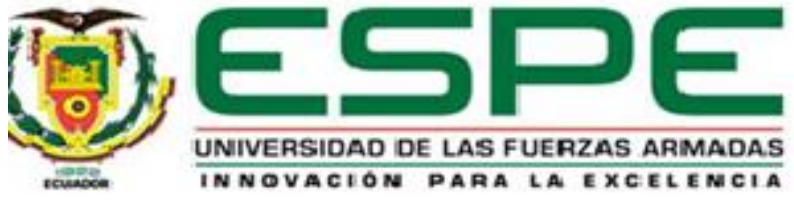

Training Systems for Control of Mobile Manipulator Robots in Augmented Reality

Herrera Balseca, Karen Alejandra y Rocha Santafé, Jessica Alexandra

Departamento de Eléctrica y Electrónica

Carrera de Ingeniería en Electrónica e Instrumentación

Artículo académico, previo a la obtención del título de Ingeniera en Electrónica e Instrumentación

Dr. Andaluz Ortiz, Víctor Hugo

12 de febrero del 2021 


\title{
Training Systems for Control of Mobile Manipulator Robots in Augmented Reality
}

\author{
Karen A. Herrera, Jessica A. Rocha, Franklin M. Silva and \\ Víctor H. Andaluz \\ Universidad de las Fuerzas Armadas ESPE \\ Sangolquí - Ecuador. \\ $\{$ kaherrera1, jarocha1, fmsilva, vhandaluz1\}@espe.edu.ec.
}

\begin{abstract}
In this article, an augmented reality application interface was developed, using a technological tool oriented towards teaching-learning that allows a mobile manipulator robot training system to be created. Focusing on the simulation of autonomous control algorithms and the implementation of animations made in the Unity 3D software, which will allow the user to interact with the real world through a mobile device where the rear camera will used to capture the image and the touch screen for visualization. It is verified that the application is intuitive and understandable for the user to operate, in order to provide the necessary knowledge of the operation and structure of the robot.
\end{abstract}

Keywords - Augmented reality; control algorithms; Unity 3D; animations.

\section{INTRODUCTION}

Immersive technology gives way to new, more useful and reasonable communication channels [1]. Through the creation of simulated artificial realities (VR), the reproduction of reality itself with visual elements (AR), and mixed reality (MR) will be the union of the two, where it is possible to conform tools that serve to design, educate, manipulate, inform, etc. [2]. The (VR) and (AR) have benefits in several sectors because of their great potential of visualization that is able to provide an extra value to the contents and data [3].

The AR begins to appear in the computer and scientific world in the early 1990s, with faster and more efficient technologies, real-time graphic representation techniques, combining the physical world with digital objects [4]; it offers high levels of natural interaction as the computer vision techniques it uses allow it to detect user interactions [5]. The term refers to a set of methods that allow virtual content such as images, 3D models superimposed in real time, where the main idea is to create an environment in which information and virtual objects are merged with the real ones [6]. The technological advances allow AR experiences are no longer only possible on portable computers, but also tend to use smart phones [7], which require features specific for processing of information and the develop the application interface [8]. As it is a mobile devicebased implementation, it has the advantage of being able to transport the interface to the place of operation [9].

The applications that are made in the AR, in the field of the industrial engineering is based on the design of the specific characteristics of an industrial environment and of several processes, which allows the user to accede to all the information in detail and to avoid damages to the plants or shutdowns of production [10]. In the field of health, it allows a more general diagnosis by having graphic interfaces of the human body; in marketing and tourism, AR begins to appear as an alternative technology because it allows the subject to interact with the real world [11], addition to developing advertising and generating profits for companies. The AR presented in education as a trend that is imposed by the development of new teaching-learning methods, which allow students to interact with new environments and promote their interest in discovering things [12] [13].

Within the area of robotics there is a field known as service robotics that introduces autonomous robots specifically designed to assist humans, offering different services based on its mechanical design, in which there are some types of robots such as air, land, water. For mobility use wheels, legs, propellers, each of them can used depending on the application and the environment to develop [14]. In the creation of the application interface, three-dimensional CAD geometry and assembly information is used [15], where the programmer can generate several $\mathrm{AR}$ scenes in $2 \mathrm{D}$ or $3 \mathrm{D}$, which allows the human being to interact with the real world through a device. Addition to assisting users during the execution of their tasks in an efficient and fast manner, saving time, money, labor and material expenses.

Another application of the AR is based on mobile robotics, for development complex simulation models, expensive hardware and a highly controlled environment are needed, therefore there is a need for robot developers to have a more flexible option to perform experiments [16].

This article focuses on the application of augmented reality technology as a tool for the simulation of a system oriented to the teaching learning of mobile manipulator robots with the aim of providing greater knowledge in the movement of the robot. In addition, autonomous control algorithms will be proposed and evaluated to perform locomotion and manipulation tasks. To this end, a mobile device will used where the rear camera allows the capture of the image and the touch screen for the visualization of the graphic interface application, in order to perform functional tests.

\section{FORMULATION OF THE PROBLEM}

In the educational field, the incorporation of technologies is more and more frequent since they bring an enrichment to the teaching-learning process; creating a unique experience at the moment of interacting in the digital environment in an individual way inciting self-discovery and acquiring skills that in the future 
will be necessary to develop in society. Focused on the technological advance since through a software the time can optimized, improving the resources being more interactive in the academic activities.

The evolution of today's technology is continually changing to keep the human being in touch with the digital or simulated world. While robotics and artificial intelligence in mobile manipulator robots facilitate the tasks of manipulating objects. The complexity in the environments that circulate are factors that affect the execution of the tasks and the tracking of the trajectories defined by the human operator, where orientation and location of the operating end in the desired point of the work area is controlled.

In this context, be proposed to develop an augmented reality application; that by means of a mobile device the information of the mobile manipulator robot can obtained through 2D and 3D markers for user interaction as shown in Fig 1.

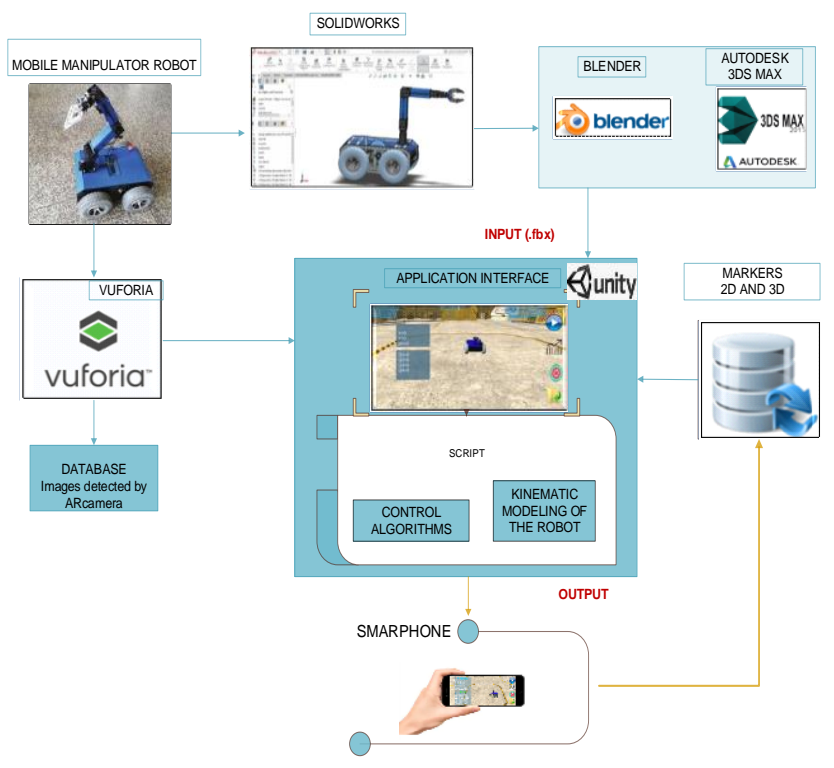

Figure 1. Outline of the proposal.

\section{SYSTEM STRUCTURE}

For the developed application, the realism of the elements is important for the design of the parametric model in the CAD software. Where four stages each with specific tasks are considered, see Fig. 2.

Layer 1. At this stage, in the SolidWorks Software develops the 3D CAD design that corresponds to each of the elements creating individual and in joint parts that interact in the assembly of both the mobile platform and the robot arm.

Layer 2. In the 3dsMax and Blender programs, it developed the creation of the animations, the $3 \mathrm{D}$ visual effects in reference to the CAD model of the assembled robot as shown in Fig. 3 (a). Where the orientation of each part is implemented, as well as its rotation and motion axes; at the end of all the necessary modifications to the CAD model of the mobile manipulator, a file is obtained with the Flimbox format (*.fbx) that is compatible with Unity's graphic engine, where the robot's animation is designed as shown in Fig. 3 (b).

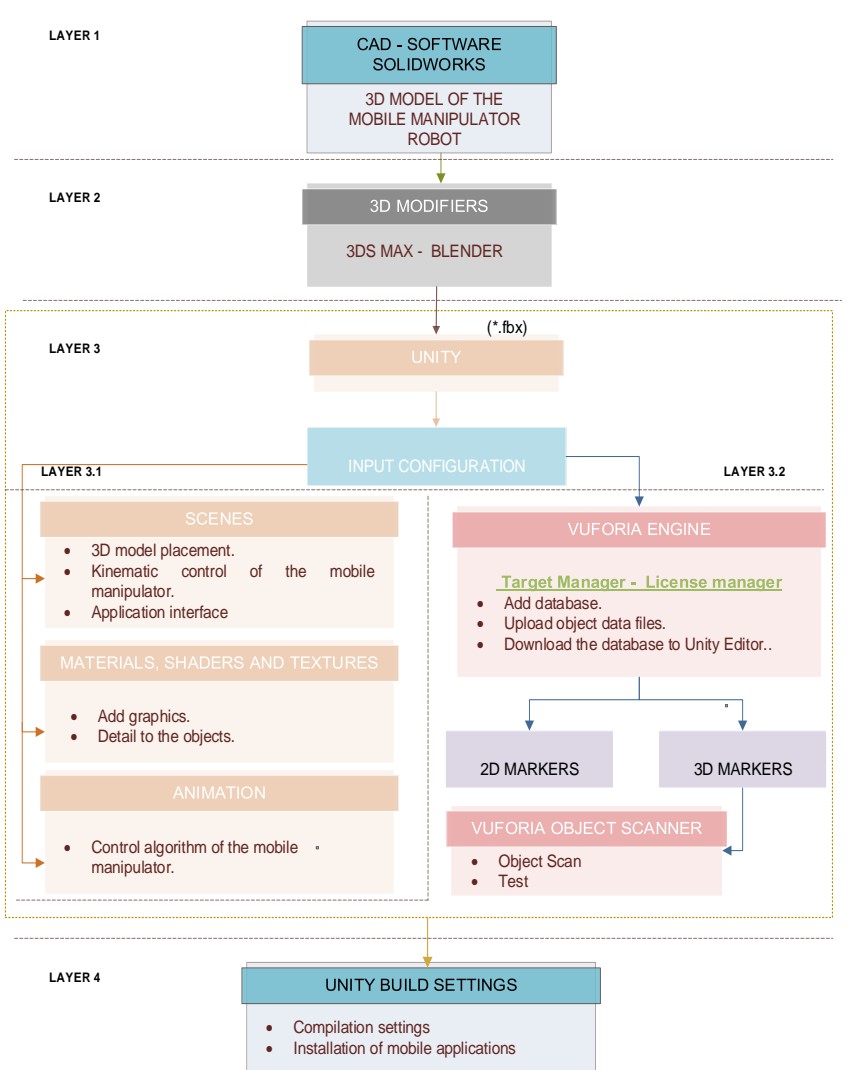

Figure 2. System structure for the creation of the application.

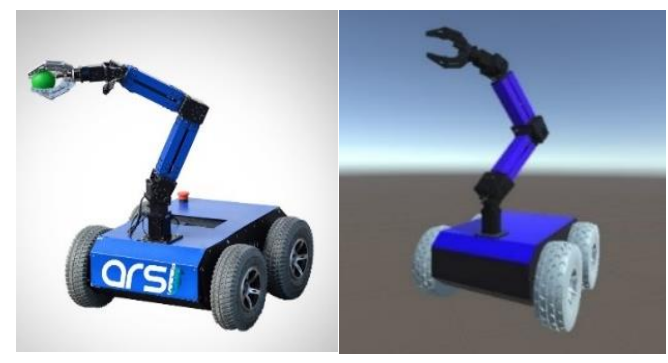

(a) Real Robot

(b) Virtual Robot

Figure 3. Robot Mobile manipulator.

Layer 3. At this stage the file (*.fbx) is imported into the Software Unity where the input settings are described and this is divided into two sections. Layer 3.1 In the Software Unity, it enters different inputs that make up four main subroutines: Subroutine 1: Interface design and application environment. Subroutine 2: Assembly of each of the objects such as the moving platform, robot arm and finally the whole robot. Subroutine 3: Characteristics of the movements that develop by means of animations. Subroutine 4: Application of control algorithms. Layer 3.2. Describes the 2D and 3D marker recognition technique. It is loaded through Vuforia Developer Portal's Target Manager; a license is obtained that allows the creation of databases and the generation of a Unity compatible file while Vuforia processes each image and generates characteristic points that give the recognition quality of said image. For the detection of 3D markers, the Vuforia Object Scanner application detects the mobile manipulator that is on a 
template, which allows obtaining the different positions of the robot, and finally it performs a recognition test of it.

Layer 4. The configuration of various presentation parameters of the application such as textures, sizes, orientation, the Android-compatible version and the package name are defined; when the configuration is finished, the application is transferred to the mobile device for installation and use, see Fig. 4.

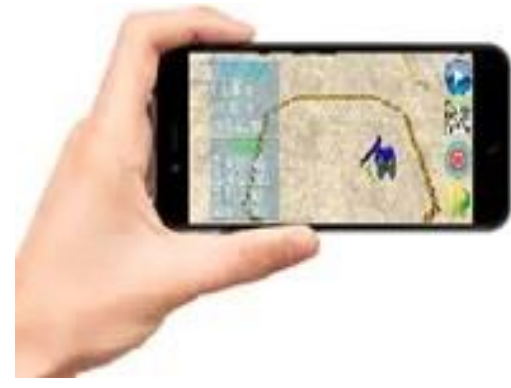

Figure 4. Application on the mobile device.

\section{MODELING AND CONTROL}

This Section presents the kinematics modeling of a manipulator robot formed by a unicycle type mobile platform and a 3DOF robotic arm, with the purpose of determining the characteristics and movement restrictions of the robot. In addition, the design of an autonomous control algorithm to execute manipulation and locomotion tasks of the mobile manipulator robot presented; and finally, it develops the stability analysis in order to assure that the control errors tend to zero asymptotically.

\section{A. Robot Modeling}

Mobile manipulator is nowadays a widespread term that refers to robots built with a robotic arm mounted on a mobile platform. This kind of system, which is usually characterized by a high degree of redundancy, combines the manipulability of a fixedbase manipulator with the mobility of a wheeled platform. Such systems allow the most usual missions of robotic systems which require both locomotion and manipulation abilities.

The kinematic model of a mobile manipulator gives the location of the end-effector $\mathbf{h}$ as a function of the robotic arm configuration and the platform location (or its operational coordinates as functions of the robotic arm's generalized coordinates and the mobile platform's operational coordinates).

$$
\begin{aligned}
f: \quad N_{a} \times M_{p} & \rightarrow M \\
\mathbf{q} & \rightarrow \mathbf{h}=f(\mathbf{q})
\end{aligned}
$$

where, $N_{a}$ is the configuration space of the robotic arm; $M_{p}$ is the operational space of the mobile platform; $\mathbf{q}$ represents the generalized coordinates of the mobile manipulator robot.

Hence, the kinematic model of a mobile manipulator gives the derivative of its end-effector location as a function of the derivatives of both the robotic arm configuration and the location of the mobile platform.

$$
\dot{\mathbf{h}}(\mathbf{t})=\mathbf{J}(\mathbf{q}) \mathbf{v}(\mathbf{t})
$$

where, $\dot{\mathbf{h}}=\left[\begin{array}{lll}\dot{h}_{x} & \dot{h}_{y} & \dot{h}_{z}\end{array}\right]^{\mathbf{T}}$ is the vector of the end-effector velocity; $\mathbf{v}=\left[\begin{array}{lllll}u & \omega & \dot{q}_{1} & \dot{q}_{2} & \dot{q}_{3}\end{array}\right]^{\mathbf{T}}$ represents the velocity vector of the mobile manipulator robot; and finally $\mathbf{J}(\mathbf{q})$ defined the Jacobian matrix has more unknowns that equation $m<n$ hence, infinite solution exists. It is worth mentioning that $m$ represents the number of dimensions of the workspace and $n$ the number of degrees of freedom of the mobile manipulator robot [17].

The Jacobian matrix represents the characteristics and movement restrictions of the mobile manipulator robot, that is, it considers the movement restrictions of the mobile platform and the robotic arm. Therefore, the main motion restriction of the mobile manipulator robot, defines that the robot can only move perpendicular to the axis connecting the wheels of the mobile platform, this restriction is defined by:

$$
\dot{x} \sin (\varphi)-\dot{y} \cos (\varphi)+a \omega=0
$$

where, $\dot{x}$ and $\dot{y}$ represent the moving platform velocities about axes $X$ y $Y$, respectively, of the inertial reference system $\langle R\rangle$; whilst $\omega$ defines the angular velocity of the mobile platform in regarding to the axis $Z$ regarding to $\langle R>$ ; and finally $a$ a constant representing the location of the robotic arm regarding to the coordinate point $(x, y)$ of the mobile platform.

\section{B. Control Algorithm}

The design of the kinematic controller is based on the kinetic model of the mobile manipulator robot (1)

$$
\dot{\mathbf{h}}(\mathbf{t})=\mathbf{J}(\mathbf{q}) \mathbf{v}(\mathbf{t})
$$

where, $\mathbf{v}$ can expressed in terms of $\dot{\mathbf{h}}$ using the pseudoinverse of the Jacobian matrix. $\mathbf{v}(\mathbf{t})=\mathbf{J}^{\#}(\mathbf{q}) \dot{\mathbf{h}}(\mathbf{t})$, where, $\mathbf{J}^{\#}(\mathbf{q})=\mathbf{W}^{-1} \mathbf{J}^{\mathbf{T}}\left(\mathbf{J W}^{-1} \mathbf{J}^{\mathbf{T}}\right)^{-\mathbf{1}}$ being $\mathbf{W}$ a positive symmetrical matrix; hence:

$$
\mathbf{v}(t)=\mathbf{W}^{-1} \mathbf{J}^{\mathbf{T}}\left(\mathbf{J W}^{-1} \mathbf{J}^{\mathbf{T}}\right)^{-1} \dot{\mathbf{h}}(t)
$$

For each moment, the mobile manipulator will carry out locomotion and object handling tasks with the least number of movements. The following control law is proposed, which it is based on a minimal norm solution.

$$
\begin{aligned}
\mathbf{v}_{\mathbf{c}}(t)= & \underbrace{\mathbf{J}^{\#}\left(\dot{\mathbf{h}}_{\mathbf{d}}+\mathbf{L}_{\mathbf{K}} \tanh \left(\mathbf{L}_{\mathbf{K}}^{-1} \mathbf{K} \tilde{\mathbf{h}}\right)\right)}_{\mathbf{T}_{1}}+\ldots \\
& \underbrace{\left(\mathbf{I}-\mathbf{J}^{\#} \mathbf{J}\right) \mathbf{L}_{\mathbf{D}} \tanh \left(\mathbf{L}_{\mathbf{D}}^{-1} \mathbf{D} \boldsymbol{\eta}\right)}_{\mathbf{T}_{2}}
\end{aligned}
$$


where, $\dot{\mathbf{h}}_{\mathbf{d}}$ is the desired velocities vector of the end-effector, $\tilde{\mathbf{h}}$ is the position error of the end-effector defined as $\tilde{\mathbf{h}}=\mathbf{h}_{\mathbf{d}} \mathbf{- h}$, while $\mathbf{h}_{\mathbf{d}}$ is positions desired vector of the end-effector; and finally $\mathbf{K}, \mathbf{L}_{\mathbf{D}}, \mathbf{L}_{\mathbf{K}}$ and $\mathbf{D}$ are positive definite diagonal gain matrices. Also, the $\mathbf{T}_{\mathbf{1}}$ term of (3) describes the primary task of the end effector which minimizes $\left\|\mathbf{v}-\mathbf{J}^{\#} \dot{\mathbf{h}}\right\|$; and $\mathbf{T}_{\mathbf{2}}$ term defines self-motion of the mobile $\left(\mathbf{I}-\mathbf{J}^{\#} \mathbf{J}\right)$ projects an arbitrary vector $\boldsymbol{\eta}$ onto the null space of the manipulator Jacobian; to avoid saturation of system velocity, the $\tanh ($.$) function is$ introduced to limit the error of $\tilde{\mathbf{h}}$ and the vector $\boldsymbol{\eta}$. For redundant manipulators, there are algorithms that, using the null space of the Jacobian, solve secondary tasks such as avoiding singularities and avoiding joint boundaries by solving a nonlinear equation or the singularities can be decoupled. In this work, the secondary objective it is considered to avoid unwanted configurations in which the end-effector cannot move or rotate in some directions in space obtaining finite velocities and do not accomplish the desired task.

\section{Stability Analysis}

The control error $\tilde{\mathbf{h}}$ is analyzed by assuming -perfect velocity tracking-, i.e., $\mathbf{v}(t) \equiv \mathbf{v}_{\mathbf{c}}(t)$; hence, replacing (3) in (1):

$$
\dot{\tilde{\mathbf{h}}}+\mathbf{L}_{\mathrm{K}} \tanh \left(\mathbf{L}_{\mathrm{K}}^{-1} \mathbf{k} \tilde{\mathbf{h}}\right)=\mathbf{0}
$$

The following Lyapunov function is considered for the stability analysis:

$$
\mathbf{V}(\tilde{\mathbf{h}})=\frac{1}{2} \tilde{\mathbf{h}}^{\mathrm{T}} \tilde{\mathbf{h}}
$$

where, temporary derivate of the system is: $\dot{\mathbf{V}}(\tilde{\mathbf{h}})=-\tilde{\mathbf{h}}^{\mathrm{T}} \mathbf{L}_{\mathbf{K}} \tanh \left(\mathbf{L}_{\mathbf{K}}^{\mathbf{1}} \mathbf{k} \tilde{\mathbf{h}}\right)<\mathbf{0}$. The closed-loop control system (4) is asymptotically stable so that the position error of the end-effector $\tilde{\mathbf{h}}(\mathbf{t}) \rightarrow \mathbf{0}$ is asymptotically, with $\mathbf{t} \rightarrow \infty$.

\section{EXPERIMENTAL RESULTS}

This Section presents the AR interface and its usefulness as a teaching-learning technological tool for handling mobile manipulator robots. To start the application, it is necessary to previously install the APK in the Smartphone; the icons used in the application, see in Table I.

TABLE I. ICONS

\begin{tabular}{ll}
\hline \multicolumn{1}{c}{ Name } \\
$\begin{array}{l}\text { Mobile } \\
\text { manipulator } \\
\text { robot }\end{array}$ \\
$\begin{array}{l}\text { 2D and 3D } \\
\text { Markers }\end{array}$ \\
Main Menu
\end{tabular}

Adjust
Return
Exit
Audio
Reset
Assembly
pobot modelling
Control
algorithms
Animation.

In the Figures 5 and 6, show the respective markers in $2 \mathrm{D}$ or $3 \mathrm{D}$. When selecting one of the two options, it is possible to access the simulation of each of the implemented control algorithms to follow a user-defined path. In each of the scenes, there are the icons of the application detailed in Table I.

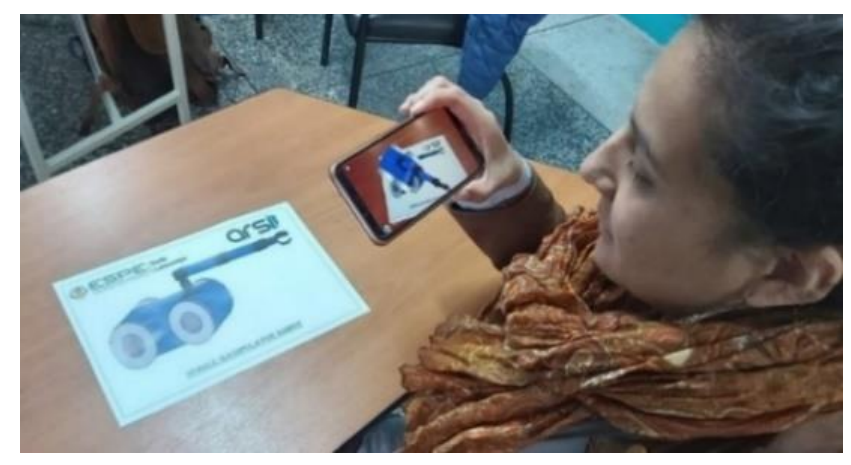

Figure 5. 2D Marker recognition.

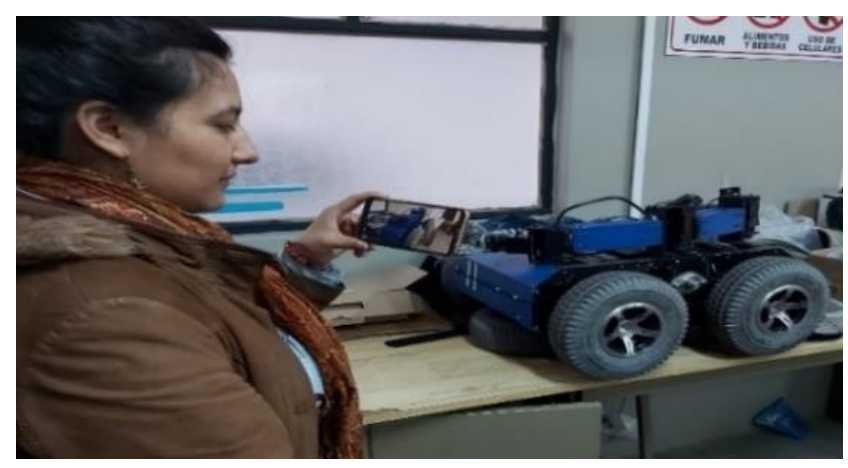

Figure 6. 3D Marker recognition.

By clicking on "Assembly", it presents the general characteristics of the mobile manipulator, and its constitution, as shown in Fig. 7. 


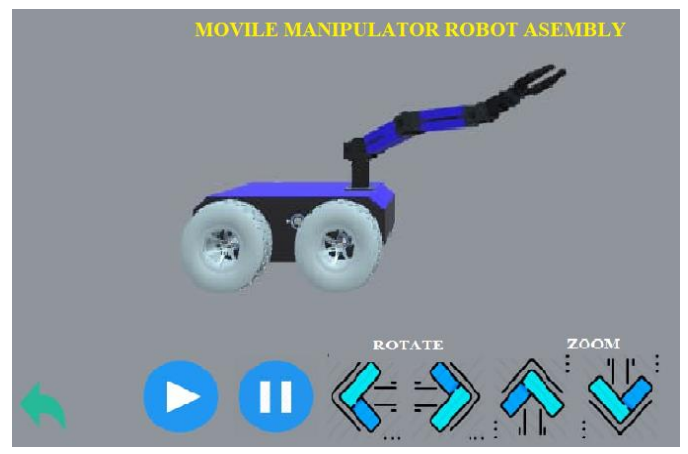

Figure 7. Assembly of the mobile manipulator robot.

By clicking on "Robot modeling parameters", it presents the general characteristics of the mobile manipulator and the constitution of its parameters to obtain the system output, as shown in Fig. 8.

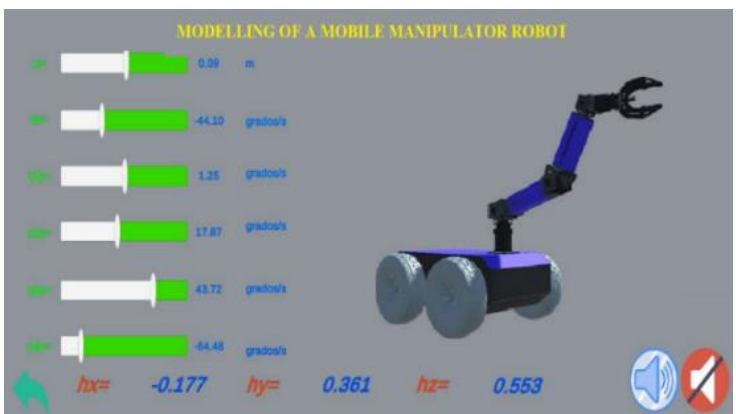

Figure 8.Parameters of the mobile manipulator

By clicking on "Control algorithms" describes the characteristics of each of the implemented algorithms such as trajectory control and the task to be performed shown in Fig. 9 and with its respective animation Fig. 10.

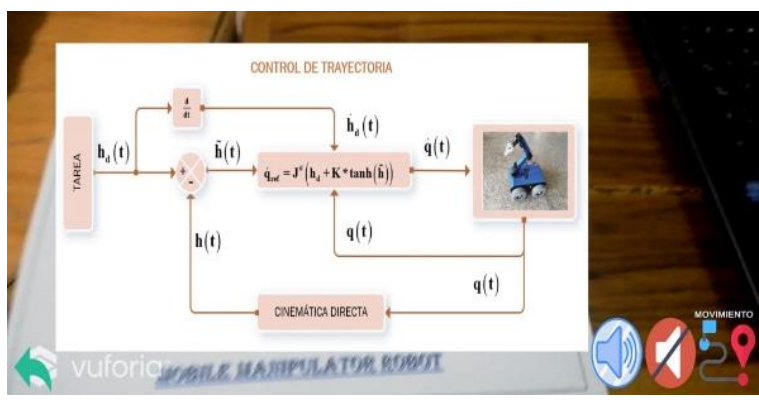

Figure 9. Description of the Trajectory algorithm.

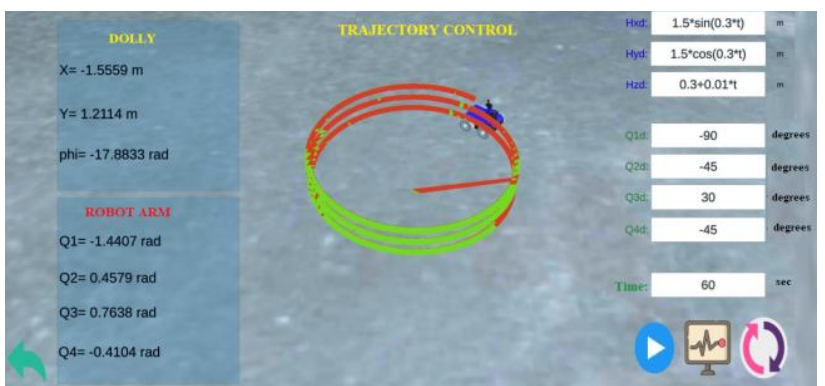

Figure 10. Moving the Mobile Manipulator Robot with the described path.
The results obtained show the stability of the path control algorithm, Fig. 11 shows the position errors $x, y, z$ in the AR application, Fig. 12 shows the output of the controller, which are the velocities that make up each of the links, Fig. 13 and 14 show the angular and linear velocities respectively of the mobile manipulator robot.

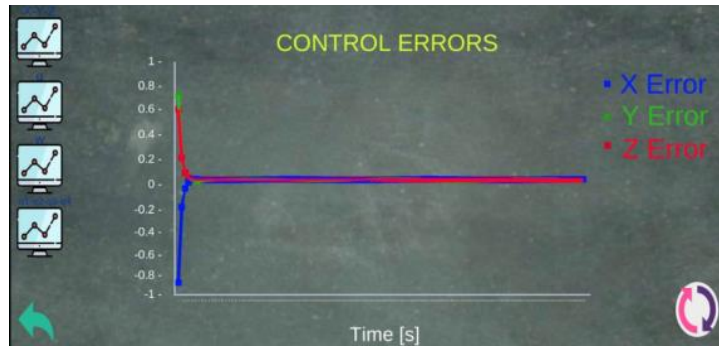

Figure 11. Control error of the mobile manipulator

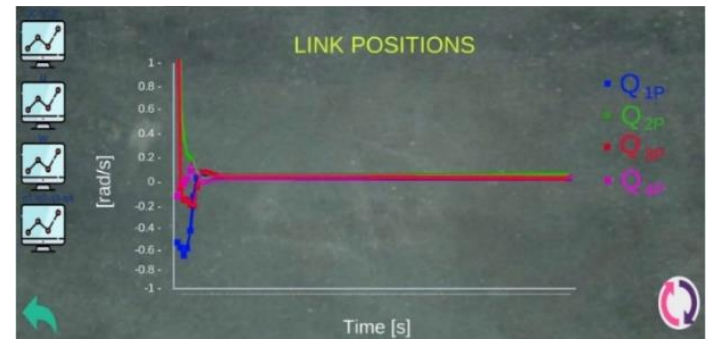

Figure 12. Joint velocity commands to the robotic arm.

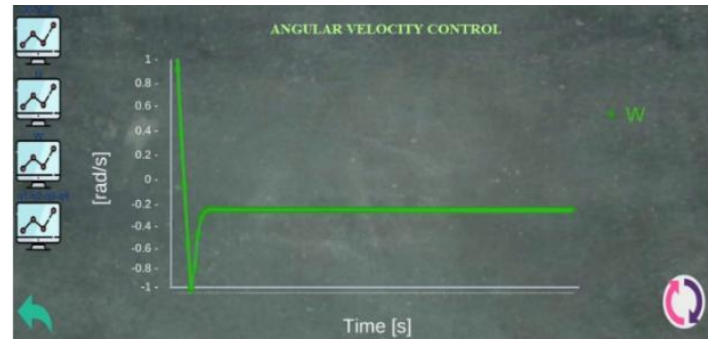

Figure 13. Angular velocity control of the moving platform

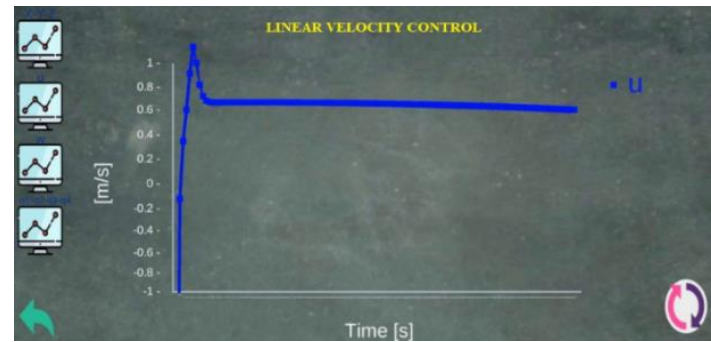

Figure 14. Linear velocity control of the moving platform.

The validation of the proposed algorithm was carried out by comparing the controller (3) implemented on the simulation of the mobile application and the AKASHA robot [18], where it is observed that the control actions make the necessary corrections so that the end-effector of the robot follows a spiral trajectory, which is parameterized in time, see Fig. 15. Fig. 16 represents the comparison of position errors $\tilde{\mathbf{h}}(t)$ between the simulation of the mobile application and the AKASHA robot. 


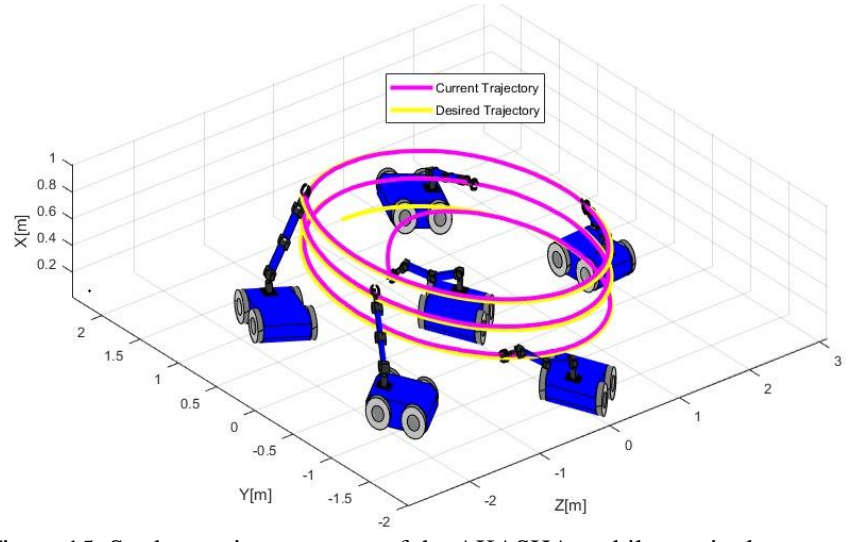

Figure 15. Stroboscopic movement of the AKASHA mobile manipulator robot.

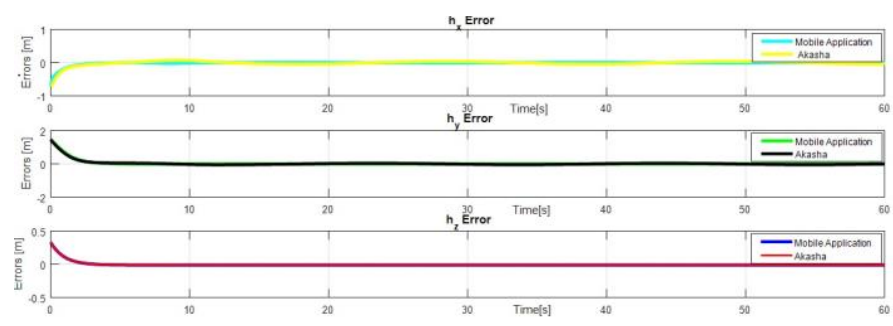

Figure 16. AKASHA control error vs. control error of the mobile application.

Finally, the results presented below indicate the validity of the usefulness of augmented reality environments for the handling of mobile manipulator robots. In the SUS method, the assessment of each item is added, where the highest range is 4 and the lowest 0 , a value of " 1 " is given if it meets the objective, but if it is not given a "0", but it focuses on measuring the reliability of the application to meet the purpose for which it was developed. For the presentation of the total results, the final score of the 10 questions, is added up and multiplied by 2.5 [19]. The survey conducted with 15 participants who are engineering students. The result will be within the range of 0 to 100 percentage as shown in Table II.

TABLE II. RESULTS OBTAINED FROM THE SURVEY

\begin{tabular}{|c|c|c|c|c|c|c|}
\hline \multirow{2}{*}{ Questions } & \multicolumn{5}{|c|}{ Score } & \multirow{2}{*}{ Operation } \\
\hline & $\mathbf{0}$ & 1 & 2 & 3 & 4 & \\
\hline $\begin{array}{l}\text { You would like to use the application } \\
\text { moderately often. }\end{array}$ & & & & 5 & 10 & 3,67 \\
\hline $\begin{array}{l}\text { It considers the implementation } \\
\text { complex and unnecessary. }\end{array}$ & 11 & 4 & & & & 3,73 \\
\hline $\begin{array}{l}\text { You believe that the use of the } \\
\text { application in augmented reality was } \\
\text { easy to use. }\end{array}$ & & & & 5 & 10 & 3,67 \\
\hline $\begin{array}{l}\text { You need a manual or assistance in } \\
\text { using the application. }\end{array}$ & 15 & & & & & 4,00 \\
\hline $\begin{array}{l}\text { You believe that the functions of the } \\
\text { application are correctly distributed } \\
\text { and integrated. }\end{array}$ & & & 3 & 1 & 11 & 3,53 \\
\hline $\begin{array}{l}\text { There is flexibility in using the } \\
\text { application. }\end{array}$ & & & 1 & 4 & 10 & 3,60 \\
\hline $\begin{array}{l}\text { He believes that people would learn } \\
\text { from using the application. }\end{array}$ & & & & 1 & 14 & 3,93 \\
\hline $\begin{array}{l}\text { When using the application, I find it } \\
\text { very difficult. }\end{array}$ & 10 & 4 & 1 & & & 3,60 \\
\hline
\end{tabular}

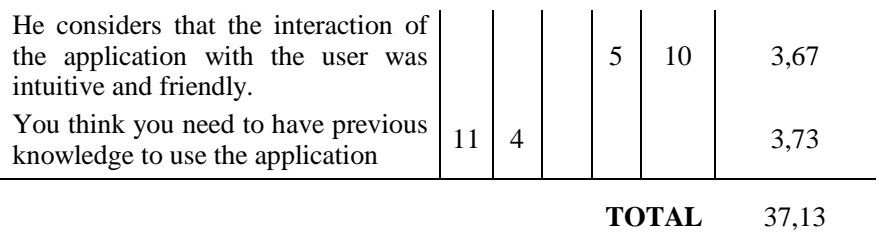

The total sum of the SUS test results is $92.83 \%$, which indicates that the utility of the application is adequate for the handling and training of mobile manipulator robots.

\section{CONCLUSIONS}

Augmented reality in a tool that contributes to teaching learning within all areas of research, as well as to the development of applications for the training of mobile manipulator robots. The application allows the user to interact with the robot and get to know each of its parts through animations. The application allows the user to interact with the robot and get to know each of its parts through animations. Visualize the robot's movement and graphics in 2D or 3D markers, in intelligent devices, with the necessary information for its operation, based on the control algorithms and the kinematic model obtained from the whole robot. Where the user can vary the inputs and obtain the desired output.

\section{ACKNOWLEDGMENT}

The authors would like to thank the Coorporación Ecuatoriana para el Desarrollo de la Investigación y Academia- CEDIA for their contribution in innovation, through the CEPRA projects, especially the project CEPRA-XIV-2020-08-RVA "Tecnologías Inmersivas Multi-Usuario Orientadas a Sistemas Sinérgicos de Enseñanza-Aprendizaje "; also the Universidad de las Fuerzas Armadas ESPE and the Research Group ARSI, for the support for the development of this work.

\section{REFERENCES}

[1] Álvarez Escobar, K. \& Álvarez Zapata, D., "Immersive technologies as a communcation strategy," from Funlam Journal of Students' Research (JSR), Medellin - Colombia, 2018.

[2] J. García, "telcel EMPRESAS," [online]. Available: https://www.telcel.com/empresas/tendencias/notas/que-son-tecnologiasinmersivas. Last accessed: 2020 February 12.

[3] Sara Arlati, Daniele Spoladore, Davide Baldassini,, "VirtualCruiseTour: An AR/VR Application," 2018.

[4] R. D. Buitrago, "State of the Art: Augmented Reality for Educational Purposes," ResearchGate, vol. 2, no. 3, pp. 1-10, 3 March 2019.

[5] Merino, C., Pino, S., Meyer, E., Garrido, J. M., \& Gallardo, F., "Augmented reality to design teaching-learning sequences in chemistry," ScienceDirect, vol. 26, no. 2, pp. 94-99, April 2015.

[6] Ierache, J., Mangiarua, N. A., Becerra, M. E., \& Igarza, S., "Framework for the Development of Augmented Reality Applications Applied to Education Games," from International Conference on Augmented Reality, Virtual Reality and Computer Graphics, June 2018.

[7] Gallardo, C., Rodríguez, S. P., Chango, I. E., Quevedo, W. X., Santana, J., Acosta, A. G., Andaluz, V. H, "Augmented Reality as a New Marketing Strategy," from International Conference on Augmented Reality, Virtual Reality and Computer Graphics, 2018.

[8] Chicaiza, E. A., Edgar, I., \& Andaluz, V. H., "Augmented reality system for training and assistance in the management of industrial equipment and instruments," from International Symposium on Visual Computing, 2018, November. 
[9] M. M. Ortega, "Remote control of the RV-M1 robot using a mobile device and Augmented Reality," 2015.

[10] Andaluz, V. H., Amaquiña, J. L., Quevedo, W. X., Mora-Aguilar, J., Castillo-Carrión, D., Miranda, R. J., \& Pérez, M. G, "Oil processes VR training," from International Symposium on Visual Computing, 2018.

[11] Acosta, A. G., Andaluz, V. H., Ortiz, J. S., Silva, F. M., Tapia, J. C., Carvajal, C. P., \& Quevedo, W. X., " e-Tourism: Governmental Planning and Management Mechanism," from International Conference on Augmented Reality, Virtual Reality and Computer Graphics, 2018, June.

[12] Montecé-Mosquera, F., Verdesoto-Arguello, A., Montecé-Mosquera, C., \& Caicedo Camposano, C., "Impacto de la realidad aumentada en la educación del siglo XXI," from European Scientific Journal, ESJ, 2017.

[13] Marín, V., "Posibilidades de uso de la Realidad Aumentada en la educación inclusiva," Facultad de Educación de Albacete, pp. 57-68, 2016.

[14] Ortiz, J. S., Erazo, A. P., Carvajal, C. P., Pérez, J. A., Proaño, L. E., \& Andaluz, V. H., "Modeling and kinematic nonlinear control of aerial mobile manipulators," In Computational Kinematics. Springer, Cham, pp. 87-95, 2018.

[15] Mourtzis, D., Zogopoulos, V., \& Vlachou, E., "Augmented reality application to support remote maintenance as a service in the robotics industry," from Procedia CIRP, 2017.

[16] Hernández, C. A. V., Martínez, A. R., \& Ceballos, N. D. M., "C Characterization of augmented reality markers for use in robotics," Review Politécnica, vol. 13, nº 25, pp. 87-102, 17 July 2017.

[17] Víctor Andaluz, Flavio Roberti, Juan Marcos Toibero, Ricardo Carell, "Adaptive unified motion control of mobile manipulators" Control Engineering Practice, Volume 20, Issue 12, Pages 1337-1352, 2012.

[18] Acosta Núñez, JF; Andaluz Ortiz, VH; González-de-Rivera Peces, G .; Garrido Salas, J., «Energy-Saver Mobile Manipulator Based on Numerical Methods,» Electronics 8, n 10, September 2019.

[19] Sauro, J., Lewis, J.R., «When designing usability questionnaires, does it hurt to be positive,» Proceedings of the SIGCHI Conference on Human Factors in Computing Systems, p. 2215-2224, May 2013. 